\title{
Simple waves and shocks in a thin film of a perfectly soluble anti-surfactant solution
}

\author{
J. J. A. Conn - B. R. Duffy - D. Pritchard $@$ \\ S. K. Wilson $\cdot$ K. Sefiane
}

Received: 12 April 2017 / Accepted: 27 June 2017 / Published online: 3 August 2017

(C) The Author(s) 2017. This article is an open access publication

\begin{abstract}
We consider the dynamics of a thin film of a perfectly soluble anti-surfactant solution in the limit of large capillary and Péclet numbers in which the governing system of nonlinear equations is purely hyperbolic. We construct exact solutions to a family of Riemann problems for this system, and discuss the properties of these solutions, including the formation of both simple-wave and uniform regions within the flow, and the propagation of shocks in both the thickness of the film and the gradient of the concentration of solute.
\end{abstract}

Keywords Anti-surfactant $\cdot$ Hyperbolic system $\cdot$ Marangoni effect $\cdot$ Riemann problems $\cdot$ Shocks $\cdot$ Simple waves

Mathematics Subject Classification 35L51 $\cdot 35 \mathrm{~L} 67 \cdot 76 \mathrm{~A} 20 \cdot 76 \mathrm{D} 45$

\section{Introduction}

When the molecules of a dissolved solute are preferentially expelled from the free surface of a solvent, the surface tension of the solution increases. Such solutes, which act in the opposite manner to better-known surfactants, may

J. J. A. Conn · B. R. Duffy · D. Pritchard · S. K. Wilson $(\bowtie)$

Department of Mathematics and Statistics, University of Strathclyde, Livingstone Tower, 26 Richmond Street, Glasgow G1 1XH, UK

e-mail: s.k.wilson@strath.ac.uk

J. J. A. Conn

e-mail: justin.conn@strath.ac.uk

B. R. Duffy

e-mail: b.r.duffy@strath.ac.uk

D. Pritchard

e-mail: david.pritchard@strath.ac.uk

K. Sefiane

School of Engineering, University of Edinburgh, The King's Buildings, Mayfield Road, Edinburgh EH9 3FB, UK

K. Sefiane

Tianjin Key Laboratory of Refrigeration Technology, Tianjin University of Commerce, Tianjin 300134, People's Republic of China

e-mail: k.sefiane@ed.ac.uk 
conveniently be referred to as "anti-surfactants". Examples of anti-surfactants include many salts, such as sodium chloride, i.e. common table salt, when added to water [1-4], water when added to short-chain alcohols [5,6], and certain resins that are included in solvent-based paints [7-10].

A fluid-dynamical model describing the behaviour of both surfactants and anti-surfactants was recently proposed by Conn et al. [11], who investigated the dependence of the surface tension $\sigma^{*}$ of the fluid on the surface excess $\Gamma^{*}$ of the solute. This key quantity describes the difference between the surface concentration $s^{*}$ and the bulk concentration $c^{*}[12,13]$. The resulting model can describe both surfactants, molecules of which accumulate preferentially at the free surface, so that $\Gamma^{*}>0$, and anti-surfactants, molecules of which preferentially accumulate in the bulk of the fluid, so that $\Gamma^{*}<0$. In particular, if the surface concentration of solute is much greater than the bulk concentration, then the classical models for surfactants [14-22] are recovered.

In their original work, Conn et al. [11] considered only the linear stability of an infinitely deep layer of fluid. In contrast, in the present work, we obtain analytical solutions to the system of nonlinear equations describing the flow of a thin film of an anti-surfactant solution. In particular, we focus on the case of a "perfectly soluble" anti-surfactant, the molecules of which never adsorb to the free surface, so that the surface concentration $s^{*}$ is identically zero. When both capillarity and diffusion effects are negligible, the system of equations is purely hyperbolic and admits exact solutions via the method of characteristics. Specifically, in the present work we address so-called "Riemann problems" in which the initial conditions for the film thickness and the gradient of the concentration of solute are piecewise constant (see, e.g. Whitham [23]). Note that the same system of equations arises in the flow of thin films of certain solvent-based paints [7-10], but that the problems we consider here have not previously been investigated.

In Sect. 2 we briefly describe the governing equations and their simplification in the relevant regime. In Sect. 3 we present solutions to "dry-bed" problems in which one side of the domain is initially dry, and then in Sect. 4 we present solutions to "wet-bed" problems in which there is fluid everywhere.

\section{Model formulation}

We consider a thin, two-dimensional film of a perfectly soluble anti-surfactant solution of constant viscosity $\mu^{*}$ and density $\rho^{*}$ lying on top of a flat substrate. (Stars denote dimensional quantities, but dimensionless quantities will be unadorned.) We assume that the typical depth of the film $H^{*}$ is much smaller than the horizontal length scale $L^{*}$, i.e. that the aspect ratio $\epsilon=H^{*} / L^{*} \ll 1$ of the film is small. Since the anti-surfactant solution is perfectly soluble, the surface concentration of solute is identically zero, and, since the film is thin, gravity effects are neglected. The dimensionless film thickness $h=h^{*} / H^{*}$ is scaled by $H^{*}$, and the bulk concentration of solute $c=c^{*} / C^{*}$ is scaled by the typical bulk concentration $C^{*}$. The velocity of the fluid is scaled to reflect the fact that flow is driven by gradients in surface tension due to gradients in the concentration of solute, i.e. by the Marangoni effect.

Adopting the natural Cartesian coordinate system and following the reduction of the model proposed by Conn et al. [11] to the thin-film regime as described by Conn [24], the governing equations for $h(x, t)$ and $c(x, t)$ are

$$
\begin{aligned}
& \frac{\partial h}{\partial t}+\frac{\partial}{\partial x}\left(\frac{1}{3 \mathrm{Ca}} h^{3} \frac{\partial^{3} h}{\partial x^{3}}+\frac{1}{2} h^{2} \frac{\partial c}{\partial x}\right)=0, \\
& h \frac{\partial c}{\partial t}+\left(\frac{1}{3 \mathrm{Ca}} h^{3} \frac{\partial^{3} h}{\partial x^{3}}+\frac{1}{2} h^{2} \frac{\partial c}{\partial x}\right) \frac{\partial c}{\partial x}-\frac{1}{\operatorname{Pe}} \frac{\partial}{\partial x}\left(h \frac{\partial c}{\partial x}\right)=0 .
\end{aligned}
$$

Here

$\mathrm{Ca}=\frac{R^{*} T^{*} \eta^{*} C^{*}}{\epsilon^{2} \sigma_{\text {solv }}^{*}}$ and $\mathrm{Pe}=\frac{H^{*} R^{*} T^{*} \eta^{*} C^{*}}{\mu^{*} D^{*}}$

are a capillary number and a Péclet number, respectively, in which $R^{*}$ is the ideal gas constant, $T^{*}$ is the (constant) temperature, $\eta^{*}$ is the notional thickness of the free surface which is taken to be of the order of a few ångströms $[11,25], \sigma_{\text {solv }}^{*}$ is the surface tension of the pure solvent, and $D^{*}$ is the diffusivity of the solute. 
When both capillarity and diffusion effects are negligible, i.e. in the limit $\mathrm{Ca} \rightarrow \infty$ and $\mathrm{Pe} \rightarrow \infty$, the full thin-film problem given by (1) and (2) reduces to

$$
\begin{aligned}
& \frac{\partial h}{\partial t}+\frac{1}{2} \frac{\partial}{\partial x}\left(h^{2} \frac{\partial c}{\partial x}\right)=0, \\
& \frac{\partial c}{\partial t}+\frac{1}{2} h\left(\frac{\partial c}{\partial x}\right)^{2}=0,
\end{aligned}
$$

which express the conservation of mass of the fluid and of the solute, respectively.

Differentiating (5) with respect to $x$ and making the substitution $\partial c / \partial x=b$, where $b(x, t)$ denotes the gradient of the concentration of solute, we obtain the equations

$$
\begin{aligned}
& \frac{\partial h}{\partial t}+\frac{1}{2} \frac{\partial}{\partial x}\left(h^{2} b\right)=0, \\
& \frac{\partial b}{\partial t}+\frac{1}{2} \frac{\partial}{\partial x}\left(h b^{2}\right)=0 .
\end{aligned}
$$

Note that, because of the Marangoni effect, a positive value of $b$ corresponds to a positive shear stress at the free surface of the film which drives the fluid to the right, and vice versa for a negative value of $b$.

The system of nonlinear equations given by (6) and (7) is purely hyperbolic, and so may be written in characteristic form with Riemann invariants $r_{ \pm}=h b^{ \pm 1}$, which are constant along the characteristic curves in the $(x, t)$-plane with slopes given by the eigenvalues $\lambda_{ \pm}=h b\left(1 \pm \frac{1}{2}\right)$. Thus

$$
\begin{gathered}
\frac{\mathrm{d}}{\mathrm{d} t}\left(r_{+}\right)=\frac{\mathrm{d}}{\mathrm{d} t}(h b)=0, \\
\frac{\mathrm{d}}{\mathrm{d} t}\left(r_{-}\right)=\frac{\mathrm{d}}{\mathrm{d} t}\left(\frac{h}{b}\right)=0
\end{gathered}
$$

on the characteristic curves given by

$$
\frac{\mathrm{d} x}{\mathrm{~d} t}=\lambda_{ \pm}=h b\left(1 \pm \frac{1}{2}\right)
$$

With the governing equations (4) and (5) written in the characteristic form (8)-(10), we are now able to solve a family of Riemann problems in which there is a discontinuity in the initial conditions for the film thickness $h$ and/or the concentration gradient $b$. Specifically, we consider situations in which an initial discontinuity separates two otherwise uniform regions in each of which $h$ and $b$ are constant. Without loss of generality, we take the initial discontinuity to be at $x=0$, and so take the initial conditions at $t=0$ to be

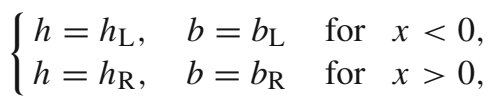

where, in general, none of the prescribed constants $h_{\mathrm{L}}, h_{\mathrm{R}}, b_{\mathrm{L}}$ and $b_{\mathrm{R}}$ are equal, and the subscripts $\mathrm{R}$ and $\mathrm{L}$ denote initial quantities to the right and to the left of the initial discontinuity, i.e. for $x>0$ and $x<0$, respectively. Note that since $b$ is initially piecewise constant, $c$ will initially be piecewise linear, and hence if $b_{\mathrm{L}}>0$ and/or $b_{\mathrm{R}}<0$ then $c$ will take unphysical negative values as $x \rightarrow-\infty$ and/or $x \rightarrow \infty$. Thus in these cases the present analysis is strictly only a local (rather than a global) one. However, as we shall show, in all cases the present analysis gives valuable insight into the surprisingly complicated dynamics that can arise from an initial discontinuity. 


\section{The "dry-bed" problem}

In this section we consider the "dry-bed" problem in which $h_{\mathrm{L}}>0$ but $h_{\mathrm{R}}=b_{\mathrm{R}}=0$, so that the region $x>0$ is initially dry.

In order to construct solutions for $h$ and $b$, we consider the characteristics in the $(x, t)$-plane given by (10). There are two characteristics emanating from any initial point $(x, 0)$, which we label as $C_{ \pm}$, corresponding to the eigenvalues $\lambda_{ \pm}$, respectively, and we use the superscripts $\mathrm{R}$ and $\mathrm{L}$ to denote characteristics emanating from the right and from the left of the initial discontinuity, i.e. from $x>0$ and $x<0$, respectively.

For $x>0$ we have $h=b \equiv 0$ at $t=0$, and so the $C_{ \pm}^{\mathrm{R}}$ characteristics coincide and are simply vertical straight lines given by

$C_{ \pm}^{\mathrm{R}}: x=x_{ \pm}^{\mathrm{R}}$

where $x_{ \pm}^{\mathrm{R}}$ is a constant that labels each of the characteristics. On the other hand, for $x<0$ we have $h=h_{\mathrm{L}}$ and $b=b_{\mathrm{L}}$ at $t=0$, and so the $C_{ \pm}^{\mathrm{L}}$ characteristics are inclined straight lines given by

$C_{+}^{\mathrm{L}}: x=\frac{3}{2} h_{\mathrm{L}} b_{\mathrm{L}} t+x_{+}^{\mathrm{L}}$,

$C_{-}^{\mathrm{L}}: x=\frac{1}{2} h_{\mathrm{L}} b_{\mathrm{L}} t+x_{-}^{\mathrm{L}}$,

where $x_{ \pm}^{\mathrm{L}}$ are constants that label each of the characteristics. Evidently, the slopes of the $C_{ \pm}^{\mathrm{L}}$ (but not the $C_{ \pm}^{\mathrm{R}}$ ) characteristics depend on the values and signs of $h_{\mathrm{L}}$ and $b_{\mathrm{L}}$. Physically, since $h$ is a thickness, we are restricted to positive values of $h_{\mathrm{L}}$, but, since $b$ is a concentration gradient, $b_{\mathrm{L}}$ can be either positive or negative, and we now consider these possibilities in turn.

3.1 The dry-bed problem with $b_{\mathrm{L}}<0$

Firstly, consider the case $b_{\mathrm{L}}<0$ in which a negative concentration gradient drives the fluid to the left. In this case, the $C_{ \pm}^{\mathrm{L}}$ characteristics have a negative slope, and hence the information carried by these characteristics propagates to the left.

Figure 1 shows the typical arrangement of the characteristics in the $(x, t)$-plane in this case. Three separate regions are identified, namely a uniform region to the left (labelled $U_{L}$ ), a uniform region to the right (labelled $U_{R}$ ), and a simple-wave region (labelled $\mathrm{SW}$ ). The dashed lines correspond to the $C_{ \pm}^{\mathrm{R}}$ characteristics in region $\mathrm{U}_{\mathrm{R}}$ and the $C_{-}^{\mathrm{L}}$ characteristics in region $\mathrm{U}_{\mathrm{L}}$, the thin solid lines correspond to the $C_{+}^{\mathrm{L}}$ characteristics in region $\mathrm{U}_{\mathrm{L}}$ and the $C_{+}$characteristics which form the expansion fan in region SW, and the thick solid lines represent the limiting characteristics which form the boundaries of region SW. We now construct the solutions that hold in each of these regions.

Region $\mathrm{U}_{\mathrm{L}}$ corresponds to the uniform region of undisturbed fluid to the left, i.e. extending to $x \rightarrow-\infty$, with the right-hand boundary of region $\mathrm{U}_{\mathrm{L}}$ being the limiting $C_{+}^{\mathrm{L}}$ characteristic, i.e. the straight line given by (13) with $x_{+}^{\mathrm{L}}=0$. The solutions for $h$ and $b$ in region $\mathrm{U}_{\mathrm{L}}$ are therefore simply

$h=h_{\mathrm{L}}, \quad b=b_{\mathrm{L}}(<0)$ for $x \leq \frac{3}{2} h_{\mathrm{L}} b_{\mathrm{L}} t(<0)$.

The corresponding solution for $c$ is obtained by integrating $\partial c / \partial x=b_{\mathrm{L}}$ with respect to $x$ and (5) with respect to $t$ to obtain

$c=c_{\mathrm{L}}+b_{\mathrm{L}} x-\frac{1}{2} h_{\mathrm{L}} b_{\mathrm{L}}{ }^{2} t$ for $x \leq \frac{3}{2} h_{\mathrm{L}} b_{\mathrm{L}} t(<0)$, 


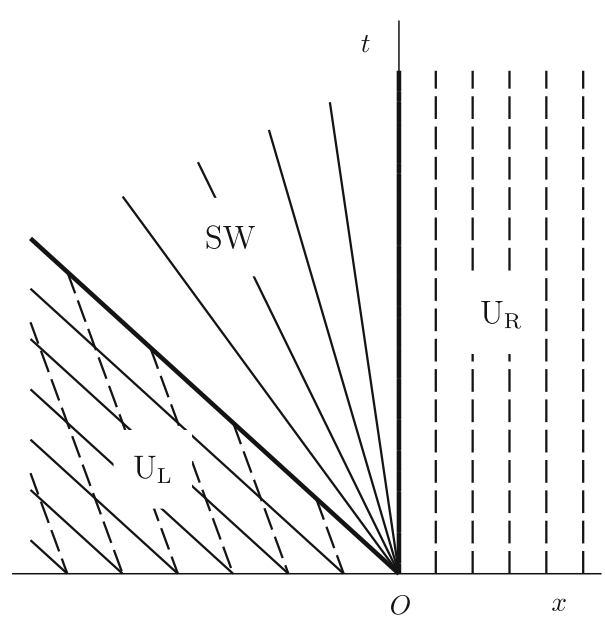

Fig. 1 The typical arrangement of the characteristics in the $(x, t)$-plane for the dry-bed problem with $b_{\mathrm{L}}<0$. Three separate regions are identified, namely a uniform region to the left (labelled $\mathrm{U}_{\mathrm{L}}$ ), a uniform region to the right (labelled $\mathrm{U}_{\mathrm{R}}$ ), and a simple-wave region (labelled SW). The dashed lines correspond to the $C_{ \pm}^{\mathrm{R}}$ characteristics in region $\mathrm{U}_{\mathrm{R}}$ and the $C_{-}^{\mathrm{L}}$ characteristics in region $\mathrm{U}_{\mathrm{L}}$, the thin solid lines correspond to the $C_{+}^{\mathrm{L}}$ characteristics in region $\mathrm{U}_{\mathrm{L}}$ and the $C_{+}$characteristics which form the expansion fan in region $\mathrm{SW}$, and the thick solid lines represent the limiting characteristics which form the boundaries of region SW

where $c_{\mathrm{L}}$ is a constant of integration.

Similarly, region $\mathrm{U}_{\mathrm{R}}$ corresponds to the uniform region with no fluid to the right of $x=0$, i.e. extending from $x=0$ to $x \rightarrow \infty$. The solutions for $h, b$ and $c$ in region $\mathrm{U}_{\mathrm{R}}$ are therefore simply

$h \equiv 0, \quad b \equiv 0, \quad c \equiv 0$ for $x \geq 0$.

The $C_{-}^{\mathrm{L}}$ characteristics emanating from region $\mathrm{U}_{\mathrm{L}}$ intersect the limiting $C_{+}^{\mathrm{L}}$ characteristic, and then enter region $\mathrm{SW}$. Since these characteristics carry the same value of $r_{-}=r_{-}^{\mathrm{L}}$ in both regions, we have

$\frac{h}{b}=\frac{h_{\mathrm{L}}}{b_{\mathrm{L}}}$ in region $\mathrm{SW}$.

Furthermore, since each $C_{+}$characteristic carries a constant value of $r_{+}$, we also have

$h b=k$ in region $\mathrm{SW}$

for some constant $k$ that is different on each $C_{+}$characteristic in region SW. Solving Eqs. (18) and (19) shows that both $h$ and $b$ are constant along any given $C_{+}$characteristic in region SW. Thus $\lambda_{+}$must be constant for any value of $k$ and, from (10), the $C_{+}$characteristics in region SW must be straight lines through the origin of the $(x, t)$-plane with slopes depending on the value of $k$. These $C_{+}$characteristics form the expansion fan in region SW shown in Fig. 1. Furthermore, since all $C_{+}$characteristics are straight lines, we may write

$\frac{\mathrm{d} x}{\mathrm{~d} t}=\frac{x}{t}=\frac{3}{2} h b$.

Solving Eqs. (18) and (20), we find the simple-wave solutions for $h$ and $b$ in region SW to be

$h=\sqrt{\frac{2 h_{\mathrm{L}} x}{3 b_{\mathrm{L}} t}}, \quad b=-\sqrt{\frac{2 b_{\mathrm{L}} x}{3 h_{\mathrm{L}} t}}(<0)$, 
(a)

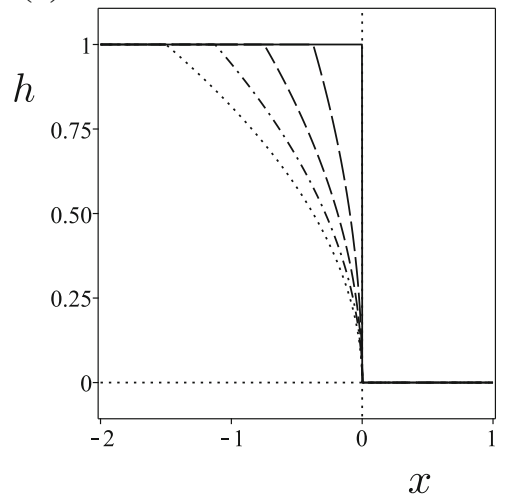

(b)

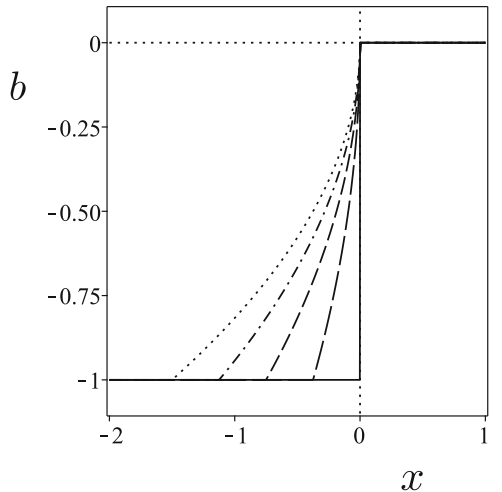

(c)

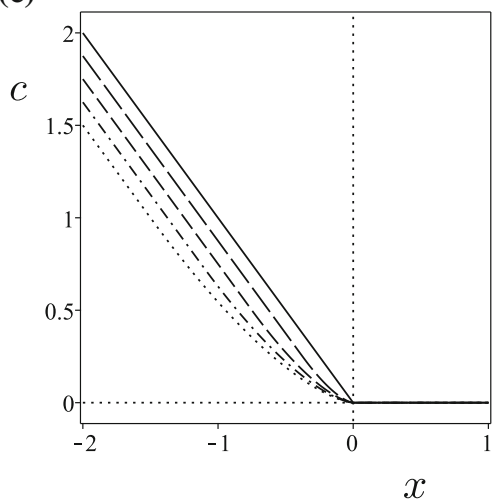

Fig. 2 Exact solutions of the dry-bed problem with $b_{\mathrm{L}}<0$ given by Eqs. (15)-(17), (21) and (22) for a $h$, b $b$ and $\mathbf{c} c$ with initial conditions (11), where $h_{\mathrm{R}}=0, b_{\mathrm{R}}=0, h_{\mathrm{L}}=1, b_{\mathrm{L}}=-1$ and $c_{\mathrm{L}}=0$, for $t=0,0.25,0.5,0.75,1$ (solid to dotted lines, respectively)

where we have chosen the signs of the square roots appearing in $h$ and $b$ appropriately.

The corresponding simple-wave solution for $c$ in region $\mathrm{SW}$ which is continuous with the solution in region $\mathrm{U}_{\mathrm{L}}$ across the limiting $C_{+}^{\mathrm{L}}$ characteristic, i.e. across $x=3 h_{\mathrm{L}} b_{\mathrm{L}} t / 2$, is

$c=c_{\mathrm{L}}+\left(\frac{2}{3}\right)^{3 / 2} \sqrt{\frac{b_{\mathrm{L}} x^{3}}{h_{\mathrm{L}} t}}$.

The presence of the arbitrary constant, namely $c_{\mathrm{L}}$, in the solution for $c$ reflects the fact that adding a uniform amount of solute to the film has no effect on the dynamics of the system, i.e. only gradients in the concentration of solute affect the behaviour of the film.

Figure 2 shows typical plots of the exact solutions given by (15)-(17), (21) and (22), and, in particular, shows the uniform solutions to the right and to the left and the simple-wave solutions that connect them. Since $b$ is always negative, the negative concentration gradient always drives the fluid to the left, advecting the solute with it. Note that, since gravity, capillarity and diffusion effects have all been neglected, there is no physical mechanism to drive the fluid rightwards, and so the initially dry region $x>0$ always remains dry.

\subsection{The dry-bed problem with $b_{\mathrm{L}}>0$}

Secondly, consider the case $b_{\mathrm{L}}>0$ in which a positive concentration gradient drives the fluid to the right. In this case, the $C_{ \pm}^{\mathrm{R}}$ characteristics, given by (12), are again vertical straight lines, but the $C_{ \pm}^{\mathrm{L}}$ characteristics, given by (13) and (14), now have a positive slope. The $C_{+}$and $C_{-}$characteristics therefore intersect at the origin of the $(x, t)$-plane, meaning that shocks form instantly (i.e. at $t=0$ ) in both $h$ and $b$ at $x=0$, and for $t>0$ these shocks propagate with some speed $\dot{x}_{\mathrm{s}}$. The speed of the shocks $\dot{x}_{\mathrm{s}}$ is determined using the Rankine-Hugoniot shock conditions [23] for this problem, namely

$\dot{x}_{\mathrm{s}} \llbracket h \rrbracket=\frac{1}{2} \llbracket h^{2} b \rrbracket$,

$\dot{x}_{\mathrm{s}} \llbracket b \rrbracket=\frac{1}{2} \llbracket h b^{2} \rrbracket$,

where the notation $\llbracket u \rrbracket$ denotes the jump in the quantity $u$ across the shock. Solving (23) and/or (24) yields

$\dot{x}_{\mathrm{s}}=\frac{1}{2} h_{\mathrm{L}} b_{\mathrm{L}}(>0)$, 
(a)

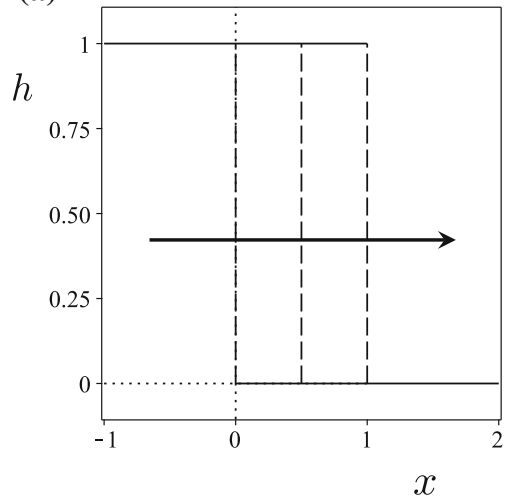

(b)

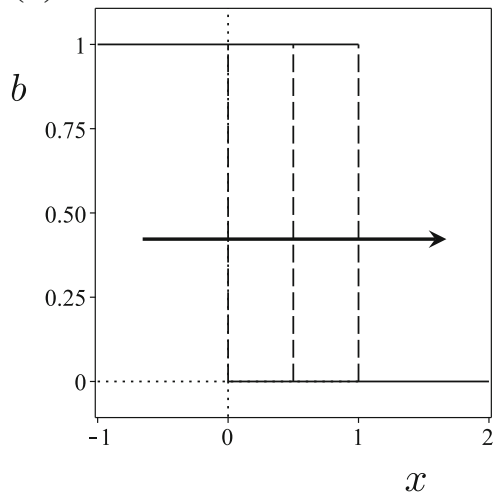

(c)

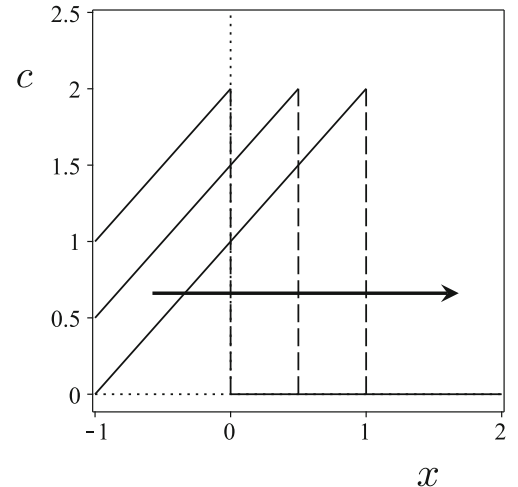

Fig. 3 Exact solutions of the dry-bed problem with $b_{\mathrm{L}}>0$ given by Eq. (26) for $\mathbf{a} h$, $\mathbf{b} b$ and $\mathbf{c} c$ with initial conditions (11), where $h_{\mathrm{R}}=0, b_{\mathrm{R}}=0, h_{\mathrm{L}}=1, b_{\mathrm{L}}=1$ and $c_{\mathrm{L}}=2$, for $t=0,1,2$. The arrows indicate the rightwards propagation of the shocks, which are indicated with dashed lines

showing that the location of the shocks is given by the limiting $C_{-}^{\mathrm{L}}$ characteristic. It is then straightforward to obtain the solutions for $h, b$ and $c$, namely

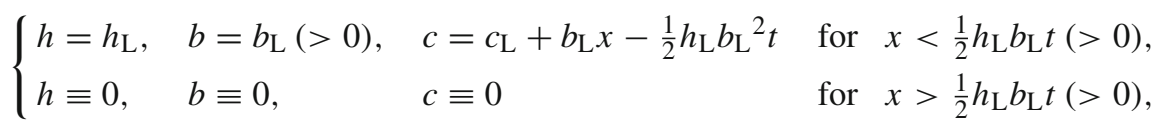

which simply represent uniform solutions for $h$ and $b$ and a linear solution for $c$, terminated by shocks that propagate rightwards at constant speed $\dot{x}_{\mathrm{s}}$ given by (25).

Figure 3 shows typical plots of the exact solutions given by (26), and, in particular, shows the rightwards propagation of the shocks. Note that, in contrast to the case $b_{\mathrm{L}}<0$ shown on Fig. 2, in this case the positive concentration gradient provides a physical mechanism that can drive the fluid rightwards into the initially dry region $x>0$.

\section{The "wet-bed" problem}

In this section we consider the "wet-bed" problem in which $h_{\mathrm{L}}>0$ and $h_{\mathrm{R}}>0$, so that there is initially fluid everywhere. Since there are now four (rather than two) free parameters, there are more cases to consider than for the dry-bed problem. However, for brevity, in the present work we consider only two of the more interesting cases, in both of which $h_{\mathrm{L}}>h_{\mathrm{R}}>0, b_{\mathrm{L}}<0$ and $b_{\mathrm{R}}<0$. In particular, in Sect. 4.1 we consider the case $h_{\mathrm{L}} b_{\mathrm{L}}<h_{\mathrm{R}} b_{\mathrm{R}}$ and show that the solution to this problem resembles that of the classical Stoker problem [26] in which a simple-wave solution continuously connects two uniform regions, the rightmost of which is connected by a shock to a further uniform region, while in Sect. 4.2 we consider the case $h_{\mathrm{L}} b_{\mathrm{L}}>h_{\mathrm{R}} b_{\mathrm{R}}$ and show that the solution to this problem consists of three uniform regions connected by two shocks. In both cases, as in the case of the dry-bed problem discussed in Sect. 3.1, since $b$ is always negative, the negative concentration gradient always drives the fluid to the left, and so the solution in the region $x>0$ always remains at its initial values.

\subsection{Wet-bed problem with $h_{\mathrm{L}} b_{\mathrm{L}}<h_{\mathrm{R}} b_{\mathrm{R}}$}

Firstly, we consider the case $h_{\mathrm{L}} b_{\mathrm{L}}<h_{\mathrm{R}} b_{\mathrm{R}}$. For $x>0$, we have $h=h_{\mathrm{R}}$ and $b=b_{\mathrm{R}}$ at $t=0$, and so the $C_{ \pm}^{\mathrm{R}}$ characteristics are straight lines given by 


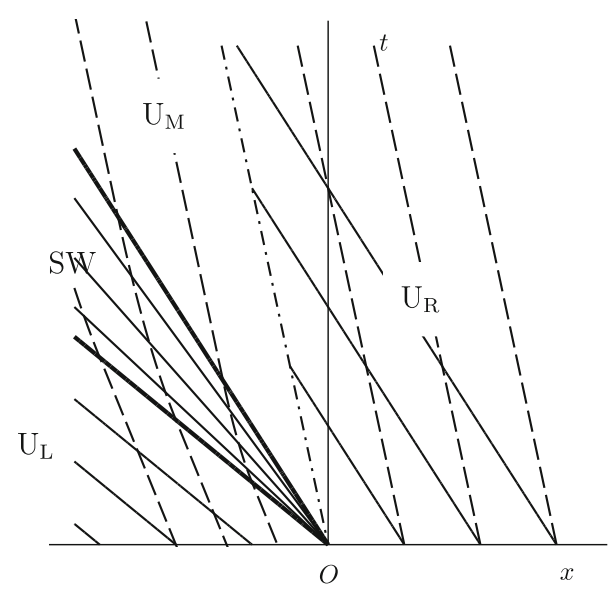

Fig. 4 The typical arrangement of the characteristics in the $(x, t)$-plane for the wet-bed problem with $h_{\mathrm{L}}>h_{\mathrm{R}}>0, b_{\mathrm{L}}<0, b_{\mathrm{R}}<0$ and $h_{\mathrm{L}} b_{\mathrm{L}}<h_{\mathrm{R}} b_{\mathrm{R}}$. In addition to two uniform regions and one simple-wave region similar to those that occur in the dry-bed problem (again labelled $U_{L}, U_{R}$ and $S W$, respectively), we also identify an additional "middle" uniform region (labelled $U_{M}$ ), not present in the dry-bed problem, that connects the simple-wave solutions in region $\mathrm{SW}$ to the uniform solutions in region $\mathrm{U}_{\mathrm{R}}$ via a shock (indicated with the dash-dot line)

$C_{+}^{\mathrm{R}}: x=\frac{3}{2} h_{\mathrm{R}} b_{\mathrm{R}} t+x_{+}^{\mathrm{R}}$,

$C_{-}^{\mathrm{R}}: x=\frac{1}{2} h_{\mathrm{R}} b_{\mathrm{R}} t+x_{-}^{\mathrm{R}}$,

where $x_{ \pm}^{\mathrm{R}}$ are constants that label each of the characteristics. For $x<0$ we have $h=h_{\mathrm{L}}$ and $b=b_{\mathrm{L}}$ at $t=0$, and so the $C_{ \pm}^{\mathrm{L}}$ characteristics are, as in the dry-bed problem, straight lines given by (13) and (14). From the forms of these characteristics, it is clear that if the constraint $h_{\mathrm{L}} b_{\mathrm{L}}<h_{\mathrm{R}} b_{\mathrm{R}}$ did not hold, then the slope of the $C_{+}^{\mathrm{R}}$ characteristics would be shallower than that of the $C_{+}^{\mathrm{L}}$ characteristics, and the $C_{+}$characteristics would therefore intersect at the origin of the $(x, t)$-plane, meaning that shocks would form instantly in both $h$ and $b$ at $x=0$. This is the situation considered in Sect. 4.2.

Figure 4 shows the typical arrangement of the characteristics in the $(x, t)$-plane for the wet-bed problem in the case $h_{\mathrm{L}} b_{\mathrm{L}}<h_{\mathrm{R}} b_{\mathrm{R}}$. In addition to two uniform regions and one simple-wave region similar to those that occur in the dry-bed problem (again labelled $\mathrm{U}_{\mathrm{L}}, \mathrm{U}_{\mathrm{R}}$ and $\mathrm{SW}$, respectively), we also identify an additional "middle" uniform region (labelled $\mathrm{U}_{\mathrm{M}}$ ), not present in the dry-bed problem, that connects the simple-wave solutions in region $\mathrm{SW}$ to the uniform solutions in region $U_{R}$ via a shock (indicated with the dash-dot line). We now construct the solutions that hold in each of these regions.

The solutions for $h, b$ and $c$ in the uniform regions $\mathrm{U}_{\mathrm{L}}$ and $\mathrm{U}_{\mathrm{R}}$ are simply

$h=h_{\mathrm{L}}, \quad b=b_{\mathrm{L}}(<0), \quad c=c_{\mathrm{L}}+b_{\mathrm{L}} x-\frac{1}{2} h_{\mathrm{L}} b_{\mathrm{L}}{ }^{2} t \quad$ for $\quad x \leq \frac{3}{2} h_{\mathrm{L}} b_{\mathrm{L}} t(<0)$

and

$h=h_{\mathrm{R}}, \quad b=b_{\mathrm{R}}(<0), \quad c=c_{\mathrm{R}}+b_{\mathrm{R}} x-\frac{1}{2} h_{\mathrm{R}} b_{\mathrm{R}}{ }^{2} t \quad$ for $\quad x \geq \frac{3}{2} h_{\mathrm{R}} b_{\mathrm{R}} t(<0)$,

respectively, where $c_{\mathrm{L}}$ and $c_{\mathrm{R}}$ are constants of integration.

As in the case of the dry-bed problem, the $C_{-}^{\mathrm{L}}$ characteristics emanating from region $\mathrm{U}_{\mathrm{L}}$ enter region $\mathrm{SW}$, and so the solutions for $h$ and $b$ in this region are precisely the same as those in the dry-bed problem given by (21) and (22), respectively. However, in contrast to the dry-bed problem, the right-hand boundary of region SW is not simply the vertical line $x=0$, but now must be found as part of the solution. Using the solutions for $h$ and $b$ in region SW, the $C_{-}^{\mathrm{L}}$ characteristics in this region satisfy 
$\frac{\mathrm{d} x}{\mathrm{~d} t}=\frac{x}{3 t}$,

and hence are given by

$t=\beta x^{3}$,

where the constant of integration $\beta(<0)$ must be negative in order that the $C_{-}^{\mathrm{L}}$ characteristics be continuous across the boundary between regions $\mathrm{U}_{\mathrm{L}}$ and $\mathrm{SW}$. After passing through region $\mathrm{SW}$, the $C_{-}^{\mathrm{L}}$ characteristics eventually intersect the boundary between regions $\mathrm{SW}$ and $\mathrm{U}_{\mathrm{M}}$, and then enter region $\mathrm{U}_{\mathrm{M}}$. Since these characteristics carry the same value of $r_{-}=r_{-}^{\mathrm{L}}$ in both regions, we have

$\frac{b}{h}=\frac{b_{\mathrm{L}}}{h_{\mathrm{L}}}$ in region $\mathrm{U}_{\mathrm{M}}$

However, there are no solutions for the uniform values of $h$ and $b$ in region $\mathrm{U}_{\mathrm{M}}$, denoted by $h_{\mathrm{M}}$ and $b_{\mathrm{M}}$, that are continuous with the solutions in region $\mathrm{U}_{\mathrm{R}}$ given by (30), and so there must be shocks in $h$ and $b$ at the boundary between regions $\mathrm{U}_{\mathrm{M}}$ and $\mathrm{U}_{\mathrm{R}}$. The uniform values of $h$ and $b$ on either side of the shocks are related by the shock conditions (23) and (24), which, along with (33), give three simultaneous (nonlinear) algebraic equations for three unknowns, namely the shock speed $\dot{x}_{\mathrm{s}}, h_{\mathrm{M}}$ and $b_{\mathrm{M}}$. Solving these equations yields

$\dot{x}_{\mathrm{s}}=\frac{1}{2} h_{\mathrm{R}} b_{\mathrm{R}}(<0)$,

showing that the location of the shocks is given by the limiting $C_{-}^{\mathrm{R}}$ characteristic, and the uniform solutions for $h$ and $b$ in region $\mathrm{U}_{\mathrm{M}}$, namely

$h_{\mathrm{M}}=\sqrt{\frac{h_{\mathrm{L}} h_{\mathrm{R}} b_{\mathrm{R}}}{b_{\mathrm{L}}}}, \quad b_{\mathrm{M}}=-\sqrt{\frac{h_{\mathrm{R}} b_{\mathrm{L}} b_{\mathrm{R}}}{h_{\mathrm{L}}}}(<0)$.

The solution for $c$ in region $\mathrm{U}_{\mathrm{M}}$, denoted by $c_{\mathrm{M}}$, which is continuous with the solution in region $\mathrm{SW}$, is

$c_{\mathrm{M}}=c_{\mathrm{L}}+\sqrt{\frac{h_{\mathrm{R}} b_{\mathrm{L}} b_{\mathrm{R}}}{h_{\mathrm{L}}}}\left(\frac{h_{\mathrm{R}} b_{\mathrm{R}} t}{2}-x\right)$.

Requiring that the solution for $c$ (but not, of course, the solutions for $h$ and $b$ ) is also continuous across the boundary between regions $\mathrm{U}_{\mathrm{M}}$ and $\mathrm{U}_{\mathrm{R}}$, i.e. across $x=\frac{1}{2} h_{\mathrm{R}} b_{\mathrm{R}} t$, shows that $c_{\mathrm{R}}=c_{\mathrm{L}}$, i.e. that, as in the dry-bed case, there is a single arbitrary constant, namely $c_{\mathrm{L}}$, in the solution for $c$ which has no effect on the dynamics of the system.

Figure 5 shows typical plots of the exact solutions for $h, b$ and $c$ given by (21), (22), (29), (30), (35) and (36). In particular, Fig. 5 shows that the solutions for both $h$ and $b$ are continuous everywhere except for shocks at the boundary between region $\mathrm{U}_{\mathrm{M}}$ and region $\mathrm{U}_{\mathrm{R}}$, which propagate leftwards into the region $x<0$ at constant speed $\dot{x}_{\mathrm{S}}$ given by (34).

\subsection{Wet-bed problem with $h_{\mathrm{L}} b_{\mathrm{L}}>h_{\mathrm{R}} b_{\mathrm{R}}$}

Secondly, we consider the case $h_{\mathrm{L}} b_{\mathrm{L}}>h_{\mathrm{R}} b_{\mathrm{R}}$, i.e. the case in which the $C_{+}$characteristics intersect at the origin of the $(x, t)$-plane, meaning that shocks form instantly in both $h$ and $b$ at $x=0$. In fact, it is immediately apparent that in this case there must be $t w o$ shocks (rather than just one shock) in both $h$ and $b$. Specifically, since the values $h_{\mathrm{L}}$, 
(a)

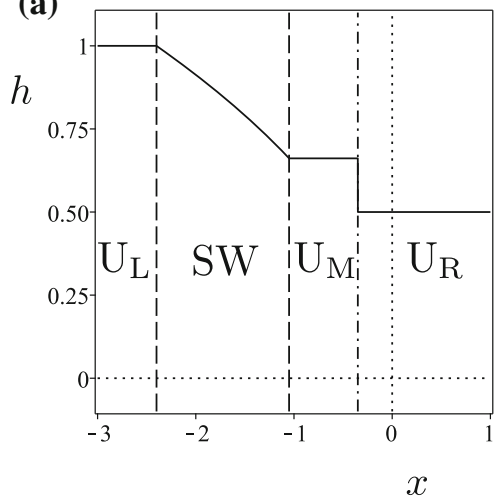

(b)

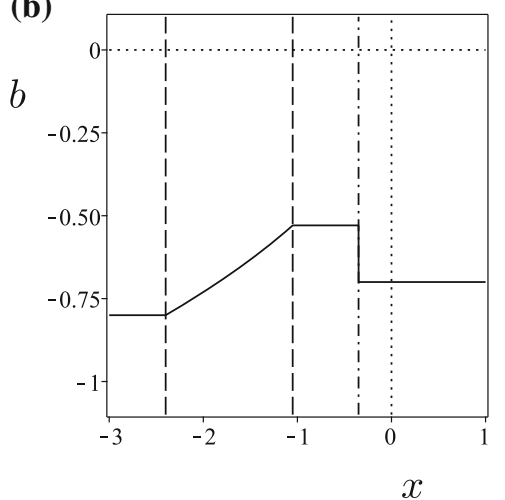

(c)

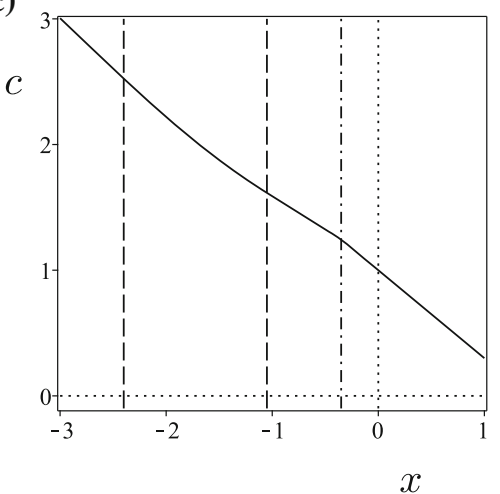

Fig. 5 Exact solutions of the wet-bed problem with $h_{\mathrm{L}}>h_{\mathrm{R}}>0, b_{\mathrm{L}}<0, b_{\mathrm{R}}<0$ and $h_{\mathrm{L}} b_{\mathrm{L}}<h_{\mathrm{R}} b_{\mathrm{R}}$ given by (21), (22), (29), (30), (35) and (36) for $\mathbf{a} h, \mathbf{b} b$ and $\mathbf{c} c$, where $h_{\mathrm{L}}=1, b_{\mathrm{L}}=-0.8, h_{\mathrm{R}}=0.5, b_{\mathrm{R}}=-0.7$ and $c_{\mathrm{L}}=1$, at $t=2$. The boundaries between the regions are indicated with dashed lines, except for the locations of the shocks at the boundary between regions $U_{M}$ and $U_{R}$, which are indicated with dash-dot lines

(a)

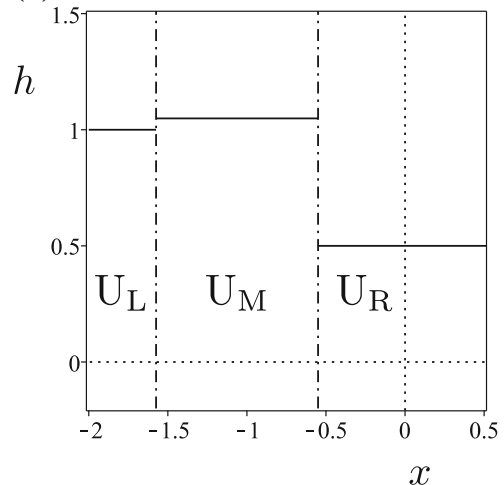

(b)

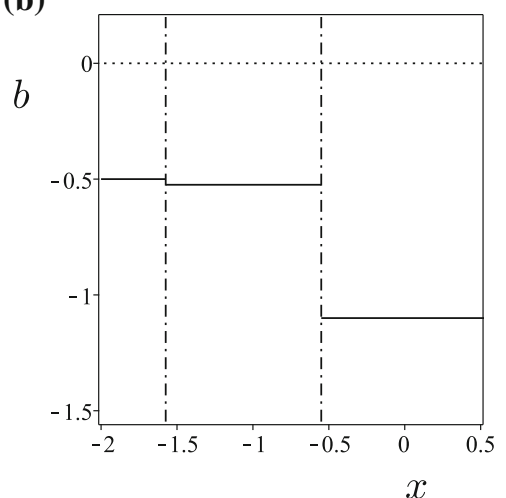

(c)

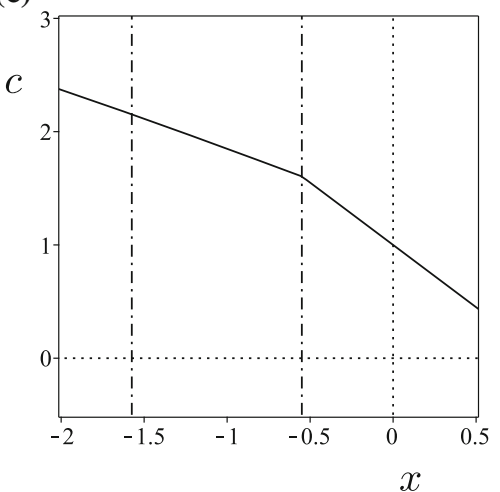

Fig. 6 Exact solutions of the wet-bed problem with $h_{\mathrm{L}}>h_{\mathrm{R}}>0, b_{\mathrm{L}}<0, b_{\mathrm{R}}<0$ and $h_{\mathrm{L}} b_{\mathrm{L}}>h_{\mathrm{R}} b_{\mathrm{R}}$ given by (29), (30), (35) and (36) for $\mathbf{a} h, \mathbf{b} b$ and $\mathbf{c} c$, where $h_{\mathrm{L}}=1, b_{\mathrm{L}}=-0.5, h_{\mathrm{R}}=0.5, b_{\mathrm{R}}=-1.1$ and $c_{\mathrm{L}}=1$, at $t=1$. The shocks at the boundaries between regions $\mathrm{U}_{\mathrm{L}}$ and $\mathrm{U}_{\mathrm{M}}$ and between regions $\mathrm{U}_{\mathrm{M}}$ and $\mathrm{U}_{\mathrm{R}}$ are indicated with dash-dot lines

$b_{\mathrm{L}}, h_{\mathrm{R}}$ and $b_{\mathrm{R}}$ are all prescribed, a single shock in both $h$ and $b$ would introduce only a single unknown (namely, the single shock speed), leading to an over-determined system, and so a second shock with a second shock speed must also occur in both $h$ and $b$.

Proceeding along the same lines as in the cases discussed previously, we find that the solution in this case consists of the uniform regions $\mathrm{U}_{\mathrm{L}}$ and $\mathrm{U}_{\mathrm{R}}$ in which the solutions for $h, b$ and $c$ are again given by (29) and (30) separated by a middle uniform region $\mathrm{U}_{\mathrm{M}}$ in which the solutions for $h, b$ and $c$ are again given by (35) and (36). Solving the appropriate shock conditions yields

$\dot{x}_{\mathrm{s}}^{\mathrm{LM}}=\frac{1}{2}\left(h_{\mathrm{L}} b_{\mathrm{L}}+h_{\mathrm{R}} b_{\mathrm{R}}-\sqrt{h_{\mathrm{L}} h_{\mathrm{R}} b_{\mathrm{L}} b_{\mathrm{R}}}\right)(<0)$

and

$\dot{x}_{\mathrm{s}}^{\mathrm{MR}}=\frac{1}{2} h_{\mathrm{R}} b_{\mathrm{R}}(<0)$, 
where $\dot{x}_{\mathrm{s}}^{\mathrm{LM}}$ and $\dot{x}_{\mathrm{s}}^{\mathrm{MR}}$ denote the speeds of the shocks at the boundaries of regions $\mathrm{U}_{\mathrm{L}}$ and $\mathrm{U}_{\mathrm{M}}$ and regions $\mathrm{U}_{\mathrm{M}}$ and $\mathrm{U}_{\mathrm{R}}$, respectively. Note that $\left|\dot{x}_{\mathrm{s}}^{\mathrm{LM}}\right|>\left|\dot{x}_{\mathrm{s}}^{\mathrm{MR}}\right|$, and so the middle region gets monotonically wider as $t$ increases, and, in particular, the shocks never collide. Furthermore, comparing the solutions in the three different regions reveals that whereas the value of $b_{\mathrm{M}}$ always lies between $b_{\mathrm{L}}$ and $b_{\mathrm{R}}$, the value of $h_{\mathrm{M}}$ is always greater than both $h_{\mathrm{L}}$ and $h_{\mathrm{R}}$, i.e. the film is always thickest in the middle region.

Figure 6 shows typical plots of the exact solutions for $h, b$ and $c$ given by (29), (30), (35) and (36). In particular, Fig. 6 shows that the solutions for both $h$ and $b$ are uniform everywhere except at the shocks, which propagate leftwards into the region $x<0$ at constant speeds $\dot{x}_{\mathrm{s}}^{\mathrm{LM}}$ and $\dot{x}_{\mathrm{s}}^{\mathrm{MR}}$ given by (37) and (38), respectively. Figure 6 also shows that the film is thickest in the middle region between the two shocks.

\section{Conclusions}

We constructed exact solutions to a family of Riemann problems that describe the dynamics of a thin film of a perfectly soluble anti-surfactant solution in the limit of large capillary and Péclet numbers. These solutions describe the formation of both simple-wave and uniform regions within the flow, and the propagation of shocks in both the thickness of the film and the gradient of concentration of solute.

While the solutions obtained in the present work are for a somewhat idealised situation, and, as discussed at the end of Sect. 2, in some cases are strictly only local solutions valid sufficiently close to the location of the initial discontinuity, they nevertheless provide an interesting analytical insight into the surprisingly complicated dynamics that can occur in this relatively simple system, and hint at even richer dynamics in the full thin-film problem given by (1) and (2). Specifically, the present solutions provide an excellent illustration of fundamental differences between surfactant and anti-surfactant dynamics: the differences between the advective transport terms for bulk and for surface concentration of solute mean that equivalent solutions are not available for thin films laden with surfactants. More pragmatically, the present solutions also provide a valuable analytical benchmark against which numerical solutions of the full thin-film problem can be validated in the future.

Acknowledgements During the course of the present work, JJAC was supported by a University of Strathclyde Postgraduate Research Scholarship, and SKW was partially supported by Leverhulme Trust Research Fellowship RF-2013-355.

Open Access This article is distributed under the terms of the Creative Commons Attribution 4.0 International License (http:// creativecommons.org/licenses/by/4.0/), which permits unrestricted use, distribution, and reproduction in any medium, provided you give appropriate credit to the original author(s) and the source, provide a link to the Creative Commons license, and indicate if changes were made.

\section{References}

1. Long FA, Nutting GC (1942) The relative surface tension of potassium chloride solutions by a differential bubble pressure method. J Am Chem Soc 64:234-243

2. Shaw DJ (1970) Introduction to colloid and surface chemistry. Butterworths, Oxford

3. Li Z, Lu BC-Y (2001) Surface tension of aqueous electrolyte solutions at high concentrations-representation and prediction. Chem Eng Sci 56:2879-2888

4. Ozdemir O, Karakashev SI, Nguyen AV, Miller JD (2009) Adsorption and surface tension analysis of concentrated alkali halide brine solutions. Miner Eng 22:263-271

5. Vázquez G, Alvarez E, Navaza JM (1995) Surface tension of alcohol + water from 20 to 50 degrees C. J Chem Eng Data 40:611-614

6. Hernández-Sánchez JF, Eddi A, Snoeijer JH (2015) Marangoni spreading due to a localized alcohol supply on a thin water film. Phys Fluids 27:032003

7. Overdiep WS (1986) The levelling of paints. Prog Org Coat 14:159-175

8. Wilson SK (1993) The levelling of paint films. IMA J Appl Math 50:149-166

9. Howison SD, Moriarty JA, Ockendon JR, Terrill EL, Wilson SK (1997) A mathematical model for drying paint layers. J Eng Math 32:377-394

10. Eres MH, Weidner DE, Schwartz LW (1999) Three-dimensional direct numerical simulation of surface-tension-gradient effects on the leveling of an evaporating multicomponent fluid. Langmuir 15:1859-1871 
11. Conn JJA, Duffy BR, Pritchard D, Wilson SK, Halling PJ, Sefiane K (2016) Fluid-dynamical model for antisurfactants. Phys Rev E 93:043121

12. Mitropoulos AC (2008) What is a surface excess? J Eng Sci Technol Rev 1:1-3

13. Langevin D (2014) Rheology of adsorbed surfactant monolayers at fluid surfaces. Annu Rev Fluid Mech 46:47-65

14. Borgas MS, Grotberg JB (1988) Monolayer flow on a thin film. J Fluid Mech 193:151-170

15. Jensen OE, Grotberg JB (1992) Insoluble surfactant spreading on a thin viscous film: shock evolution and film rupture. J Fluid Mech 240:259-288

16. Jensen OE, Grotberg JB (1993) The spreading of heat or soluble surfactant along a thin liquid film. Phys Fluids A 5:58-68

17. Matar OK, Troian SM (1999) The development of transient fingering patterns during the spreading of surfactant coated films. Phys Fluids 11:3232-3246

18. Edmonstone BD, Matar OK, Craster RV (2004) Flow of surfactant-laden thin films down an inclined plane. J Eng Math 50:141-156

19. Warner MRE, Craster RV, Matar OK (2004) Fingering phenomena created by a soluble surfactant deposition on a thin liquid film. Phys Fluids 16:2933-2951

20. Levy R, Shearer M (2006) The motion of a thin liquid film driven by surfactant and gravity. SIAM J Appl Math 66:1588-1609

21. Levy R, Shearer M, Witelski TP (2007) Gravity-driven thin liquid films with insoluble surfactant: smooth traveling waves. Eur J Appl Math 18:679-708

22. Escher J, Hillairet M, Laurencot P, Walker C (2012) Weak solutions to a thin film model with capillary effects and insoluble surfactant. Nonlinearity 25:2423-2441

23. Whitham GB (1974) Linear and Nonlinear Waves. Wiley, New York

24. Conn JJA (2017) Stability and dynamics of anti-surfactant solutions. Ph.D. thesis, Department of Mathematics and Statistics, University of Strathclyde, Glasgow

25. Chang C-H, Franses EI (1995) Adsorption dynamics of surfactants at the air/water interface: a critical review of mathematical models, data, and mechanisms. Colloids Surf A 100:1-45

26. Stoker JJ (1957) Water Waves. Interscience Publishers Inc, New York 